\title{
Sequencing and analysis of 10,967 full-length cDNA clones from Xenopus laevis and Xenopus tropicalis reveals post-tetraploidization transcriptome remodeling
}

Ryan D. Morin, ${ }^{1}$ Elbert Chang, ${ }^{1}$ Anca Petrescu, ${ }^{1}$ Nancy Liao, ${ }^{1}$ Malachi Griffith, ${ }^{1}$ Robert Kirkpatrick, ${ }^{1}$ Yaron S. Butterfield, ${ }^{1}$ Alice C. Young, ${ }^{3}$ Jeffrey Stott, ${ }^{1}$ Sarah Barber, ${ }^{1}$ Ryan Babakaiff, ${ }^{1}$ Mark C. Dickson, ${ }^{2}$ Corey Matsuo, ${ }^{1}$ David Wong, ${ }^{1}$ George S. Yang, ${ }^{1}$ Duane E. Smailus, ${ }^{1}$ Keith D. Wetherby, ${ }^{3}$ Peggy N. Kwong, ${ }^{3}$ Jane Grimwood, ${ }^{2}$ Charles P. Brinkley III, ${ }^{3}$ Mabel Brown-John, ${ }^{1}$ Natalie D. Reddix-Dugue, ${ }^{3}$ Michael Mayo, ${ }^{1}$ Jeremy Schmutz, ${ }^{2}$ Jaclyn Beland, ${ }^{1}$ Morgan Park, ${ }^{3}$ Susan Gibson, ${ }^{1}$ Teika Olson, ${ }^{1}$ Gerard G. Bouffard, ${ }^{3}$ Miranda Tsai, ${ }^{1}$ Ruth Featherstone, ${ }^{1}$ Steve Chand, ${ }^{1}$ Asim S. Siddiqui, ${ }^{1}$ Wonhee Jang, ${ }^{7}$ Ed Lee, ${ }^{7}$ Steven L. Klein, ${ }^{6}$ Robert W. Blakesley, ${ }^{3}$ Barry R. Zeeberg, ${ }^{4}$ Sudarshan Narasimhan, ${ }^{9}$ John N. Weinstein, ${ }^{4}$ Christa Prange Pennacchio, ${ }^{8}$ Richard M. Myers, ${ }^{2}$ Eric D. Green, ${ }^{3}$ Lukas Wagner, ${ }^{7}$ Daniela S. Gerhard, ${ }^{5}$ Marco A. Marra, ${ }^{1}$ Steven J.M. Jones, ${ }^{1}$ and Robert A. Holt ${ }^{1,10}$

\begin{abstract}
${ }^{1}$ British Columbia Genome Sciences Centre, BCCA, Vancouver, BC V5Z 1 L3 Canada; ${ }^{2}$ Stanford Human Genome Center and Department of Genetics, Stanford University School of Medicine, Stanford, California 94305, USA; ${ }^{3}$ NIH Intramural Sequencing Center, National Human Genome Research Institute, ${ }^{4}$ Genomics and Bioinformatics Group, Laboratory of Molecular Pharmacology, ${ }^{5}$ National Cancer Institute, and ${ }^{6}$ National Institute of Child Health and Human Development, National Institutes of Health, Bethesda, Maryland 20892, USA; ${ }^{7}$ National Center for Biotechnology Information, National Library of Medicine, Bethesda, Maryland 20894, USA; ${ }^{8}$ The I.M.A.G.E Consortium, Biology and Biotechnology Research Program, Lawrence Livermore National Laboratory, Livermore, California 94550, USA; ${ }^{9} 2$ SRA International, Fairfax, Virginia 22033, USA
\end{abstract}

\begin{abstract}
Sequencing of full-insert clones from full-length cDNA libraries from both Xenopus laevis and Xenopus tropicalis has been ongoing as part of the Xenopus Gene Collection Initiative. Here we present 10,967 full ORF verified cDNA clones (8049 from X. laevis and 2918 from X. tropicalis) as a community resource. Because the genome of X. laevis, but not X. tropicalis, has undergone allotetraploidization, comparison of coding sequences from these two clawed (pipid) frogs provides a unique angle for exploring the molecular evolution of duplicate genes. Within our clone set, we have identified 445 gene trios, each comprised of an allotetraploidization-derived $X$. laevis gene pair and their shared $X$. tropicalis ortholog. Pairwise $d_{\mathrm{N}} / d_{\mathrm{s}}$, comparisons within trios show strong evidence for purifying selection acting on all three members. However, $d_{\mathrm{N}} / d_{\mathrm{S}}$ ratios between $X$. laevis gene pairs are elevated relative to their $X$. tropicalis ortholog. This difference is highly significant and indicates an overall relaxation of selective pressures on duplicated gene pairs. We have found that the paralogs that have been lost since the tetraploidization event are enriched for several molecular functions, but have found no such enrichment in the extant paralogs. Approximately $14 \%$ of the paralogous pairs analyzed here also show differential expression indicative of subfunctionalization.
\end{abstract}

[Supplemental material is available online at www.genome.org. The sequence data from this study have been submitted to GenBank under accession nos. BC040971-BC100665 (not exclusively). Keyword MGC and organism Xenopus will be required to get only the XGC sequences in the range.]

Xenopus laevis (the African claw-toed frog) has long been a preferred model organism among developmental biologists. Features such as ease of maintenance, oocyte size and number, and

The content of this publication does not necessarily reflect the views or policies of the U.S. Department of Health and Human Services, nor does mention of trade names, commercial products, or organization imply endorsement by the U.S. Government.

${ }^{10}$ Corresponding author.

E-mail rholt@bcgsc.ca; fax (604) 877-6085.

Article published online before print. Article and publication date are at http:// www.genome.org/cgi/doi/10.1101/gr.4871006. an easily manipulated reproductive system make it an ideal organism for the study of early embryonic development (De Sa and Hillis 1990). Studies of embryonic development in Xenopus have provided insights into many salient aspects of vertebrate development that would be difficult to study in other vertebrate systems (Gilchrist et al. 2004). However, the study of Xenopus genetic material is difficult because of an allotetraploidization event in the Xenopus lineage $\sim 30$ million years ago (Mya) (Bisbee et al. 1977; Evans et al. 2004) that generated a more complex genome in all extant Xenopus species except for Xenopus tropica- 
lis, whose genome remains diploid (Graf and Kobel 1991; Hirsch et al. 2002). With a less complex genome as well as a shorter generation time, $X$. tropicalis is more amenable to genetic manipulation and has become the preferred Xenopus species for genetic analyses (Hirsch et al. 2002).

Several groups have performed large-scale EST studies on libraries from various tissues from both $X$. laevis and $X$. tropicalis (Klein et al. 2002; Blackshear et al. 2001; Gilchrist et al. 2004). However, for analysis of transcripts and gene structures, the quality of data and coverage provided by EST reads can be limiting. Sequence-verified full-length cDNA clones are more informative and have a higher sequence quality standard. Here we report the full open reading frame (ORF) sequencing and coding DNA segment (CDS) analysis of 10,967 Xenopus full-length cDNA clones (8049 from X. laevis and 2918 from $X$. tropicalis). These clones are from libraries that were constructed using RNA from numerous tissues and whole animals in various developmental stages. We expect that these clones and their verified full ORF sequences will be a valuable resource for the community. Furthermore, the availability of full ORF sequences for a large set of Xenopus clones provides a unique opportunity to study molecular evolution in the context of allotetraploidization. The putative $X$. laevis ancestral allotetraploidization event created a full set of paralogs, each from one of the parent species involved in the mating (Graf and Kobel 1991). The redundancy of the resultant tetraploid X. laevis genome has, in theory, afforded this species greater freedom to accumulate mutations that may otherwise be deleterious in a diploid genome, such as that of $X$. tropicalis. In the present study, carefully defining gene trios (gene sets comprised of the two allotetraploidization-derived $X$. laevis paralogs and their shared $X$. tropicalis ortholog) has allowed us to distinguish paralogs arising from genome duplication from paralogs arising by ordinary within-species gene family expansion. We focus our initial analysis, presented here, on detecting signatures of purifying and positive selection, and on exploring the evolution of tissue-specific gene expression.

\section{Results}

$X$. laevis and $X$. tropicalis cDNA libraries (Supplemental Tables S1 and S2) were end-sequenced by the National Intramural Sequencing Center, Washington University Genome Sequencing Center, and Agencourt Bioscience Corporation (Gerhard et al. 2004). Candidate full ORF clones were selected as previously described (Klein et al. 2002; Gerhard et al. 2004). Each candidate full ORF clone was fully sequenced to a consensus phred score of no less than 30 (Ewing and Green 1998) at each consensus position by either transposon insertion or primer walking as previously described (Wilson and Mardis 1997; Gordon et al. 1998; Butterfield et al. 2002; Strausberg et al. 2002; Yang et al. 2005). Coding DNA segment (CDS) annotation of the full insert sequences was performed as previously described (Gerhard et al. 2004). Distinct from previous MGC projects, however, we took a second approach to clone selection in an attempt to identify clones that might encode either amphibian-specific proteins or proteins too weakly conserved at the $\mathrm{N}$ terminus to be identified by comparison with proteins from other organisms. The technique we used (see Methods) assumes that a stronger conservation will be observed in the CDS than in the $5^{\prime}$-untranslated region (UTR) of paralogous genes. Most of the clones identified by this technique $(\sim 80 \%)$ were also identified by comparison with proteins from other species. The full-length sequences identified by this method are listed in Table 1 . While there are seven proteins with no significant hits $\left(E<10^{-10}\right)$ in any mammalian protein, there are also six proteins with alignment scores more than two standard deviations below the mean value to the most closely related human protein (mean $73 \%, 13.8 \%$ standard deviation). The small number of novel proteins identified as well as the overlap with proteins identified from protein or mRNA comparison suggest that there are few proteins present in this cDNA collection that are structurally distinct from previously identified proteins.

Following full insert sequencing of candidate clones, only those clones with verified complete ORFs were given an XGC (Xenopus Gene Collection) identifier. These 10,967 clones (8049 from X. laevis and 2918 from X. tropicalis) are considered the core group of XGC clones and are the basis of the analysis presented here. All clone sequences have been submitted to GenBank, and the physical clones are available through the IMAGE distribution network.

From the set of 10,967 clones, we identified 445 distinct gene trios for analysis (see Methods). Again, a trio is a gene set comprised of the two allotetraploidization-derived $X$. laevis paralogs and their shared $X$. tropicalis ortholog. To explore the signature of selection between $X$. laevis and $X$. tropicalis, we applied the $d_{\mathrm{N}} / d_{\mathrm{S}}$ test using the maximum likelihood method of Yang and Nielsen available as the codeml component of the PAML software package (PAML software release 3.14) (Yang et al. 1997). This method allows inference of evolutionary selection for mutations using the ratio of nonsynonymous $\left(d_{\mathrm{N}}\right)$ to synonymous $\left(d_{\mathrm{S}}\right) \mathrm{mu}$ tations in the coding DNA sequence of a phylogeny of homologous genes. In general, a $d_{\mathrm{N}} / d_{\mathrm{S}}$ ratio $(\omega)>1$ is evidence for positive selection acting to modify the function of a gene (Thornton and Long 2002; Zhang et al. 2002), whereas an $\omega$ significantly $<1$ suggests negative or purifying selection where functional constraint on the gene product has restricted the amount of nonsynonymous mutation. None of the pairwise comparisons between $X$. laevis paralogs or between each $X$. laevis gene and its $X$. tropicalis ortholog resulted in an $\omega$ significantly $>1$ (Fig. 1), suggesting that purifying selection has continued to act on $X$. laevis genes duplicated by allotetraploidization, and that in general

Table 1. X. laevis genes identified without protein comparison

\begin{tabular}{lc}
\hline $\begin{array}{l}\text { Xenopus laevis } \\
\text { accession }\end{array}$ & $\begin{array}{c}\text { Similarity to closest } \\
\text { Homo sapiens protein }\end{array}$ \\
\hline BC081278 & $42 \%$ \\
BC080430 & $40 \%$ \\
BC079813 & $25 \%$ \\
BC084980 & - \\
BC079815 & $35 \%$ \\
BC082713 & $35 \%$ \\
BC079817 & $44 \%$ \\
BC086299 & $35 \%$ \\
BC079818 & - \\
BC079819 & - \\
BC097879 & - \\
BC081276 & - \\
BC097923 & - \\
BC077645 & - \\
\hline
\end{tabular}

Summary of the $X$. laevis genes with no clear ortholog in $H$. sapiens. These clones were selected for sequencing by the method described, which does not rely on sequence similarity but, rather, conservation in the $5^{\prime}$ UTR. 


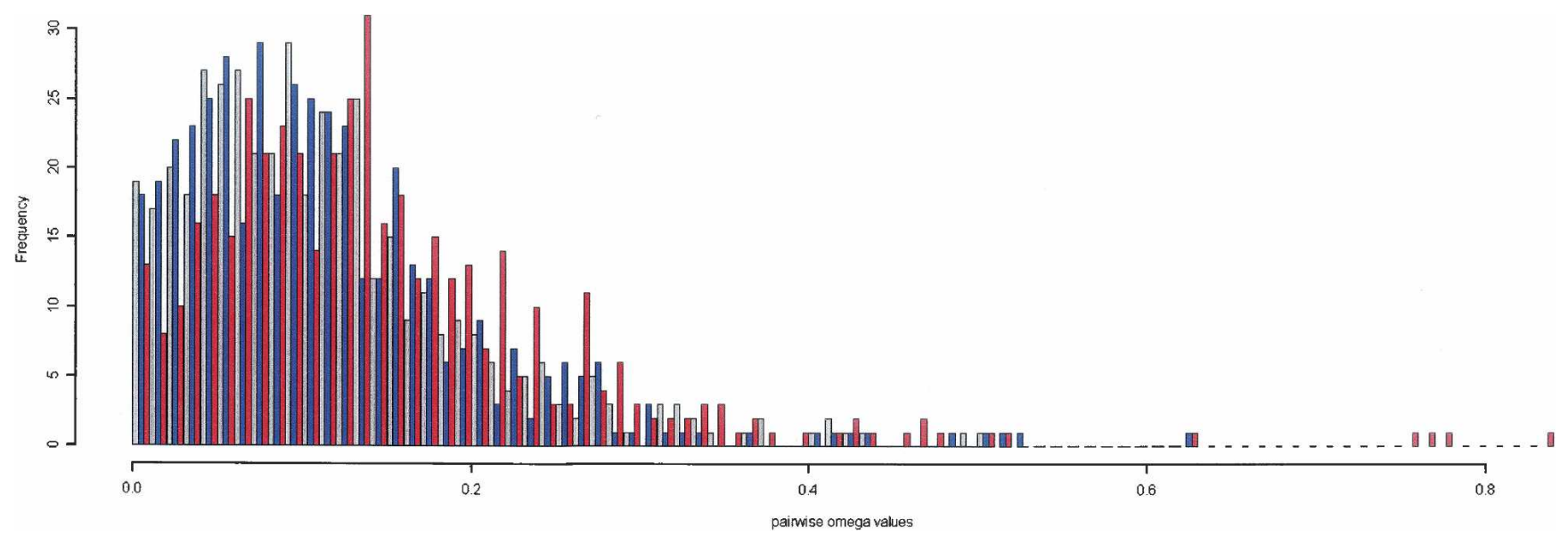

Figure 1. Frequencies of $d_{N} / d_{S}$ ratio $(\omega)$ for pairwise comparisons between $X$. laevis and $X$. tropicalis genes. The distribution of $d_{N} / d_{S}$ from pairwise comparisons of genes within gene trios is shown. $\omega$ s from $X$. laevis paralog pairs (shown in red) indicate a weaker selective constraint than the $\omega$ obtained from the comparisons of $X$. laevis paralogs with their $X$. tropicalis ortholog (shown in gray and blue). The $\omega s$ from both paralog-ortholog pairs follow a similar distribution with a lower median than the $\omega$ obtained from the paralogs in each trio $\left(P=2.184 \times 10^{-7}\right)$.

both copies have retained function. Interestingly, taken together, $\omega$ s from pairwise comparisons between $X$. laevis paralogs are significantly larger (Kruskal-Wallis rank sum test, $P=2.184 \times 10^{-7}$ ) than either of the $\omega$ s from pairwise comparisons between each $X$. laevis gene and $X$. tropicalis paralog. These observations suggest that overall there has been relaxation of selective pressures on $X$. laevis duplicated gene pairs, allowing them more freedom to accumulate nonsynonymous substitutions.

Pairwise $d_{\mathrm{S}}$ estimates provide a relative measure of time since divergence of homologous genes and give an independent estimate of the topology of our defined trios. As expected, in all of the gene trios, the $d_{\mathrm{S}}$ between the $X$. laevis paralogs is lower than both $d_{\mathrm{s}}$ s between each $X$. laevis paralog and its $X$. tropicalis ortholog. The trend (Fig. 2) shows a peak representing the divergence between the two species (blue and gray curves, me- dian $=0.2915)$ and a peak representing the tetraploidization within the $X$. laevis genome (red curve, median $=0.2039$ ). We calculated the effective number of codons (ENC) for all clones in this study using the codonW program (http://codonw. sourceforge.net) and did not see any evidence for an effect of codon bias on synonymous substitution rates.

To identify the function of genes for which duplicate copies are preferentially retained or lost after tetraploidization, we explored the Gene Ontology (GO) representation of X. laevis genes with and without paralogs. We used the High-Throughput GoMiner tool (http://discover.nci.nih.gov/gominer/htgm.jsp) (Zeeberg et al. 2005) to search for GO categories overrepresented in the clones with either active paralogs in X. laevis or no evidence for extant paralogs (see Methods). Several categories are enriched in the set of genes with no evidence for an extant para-

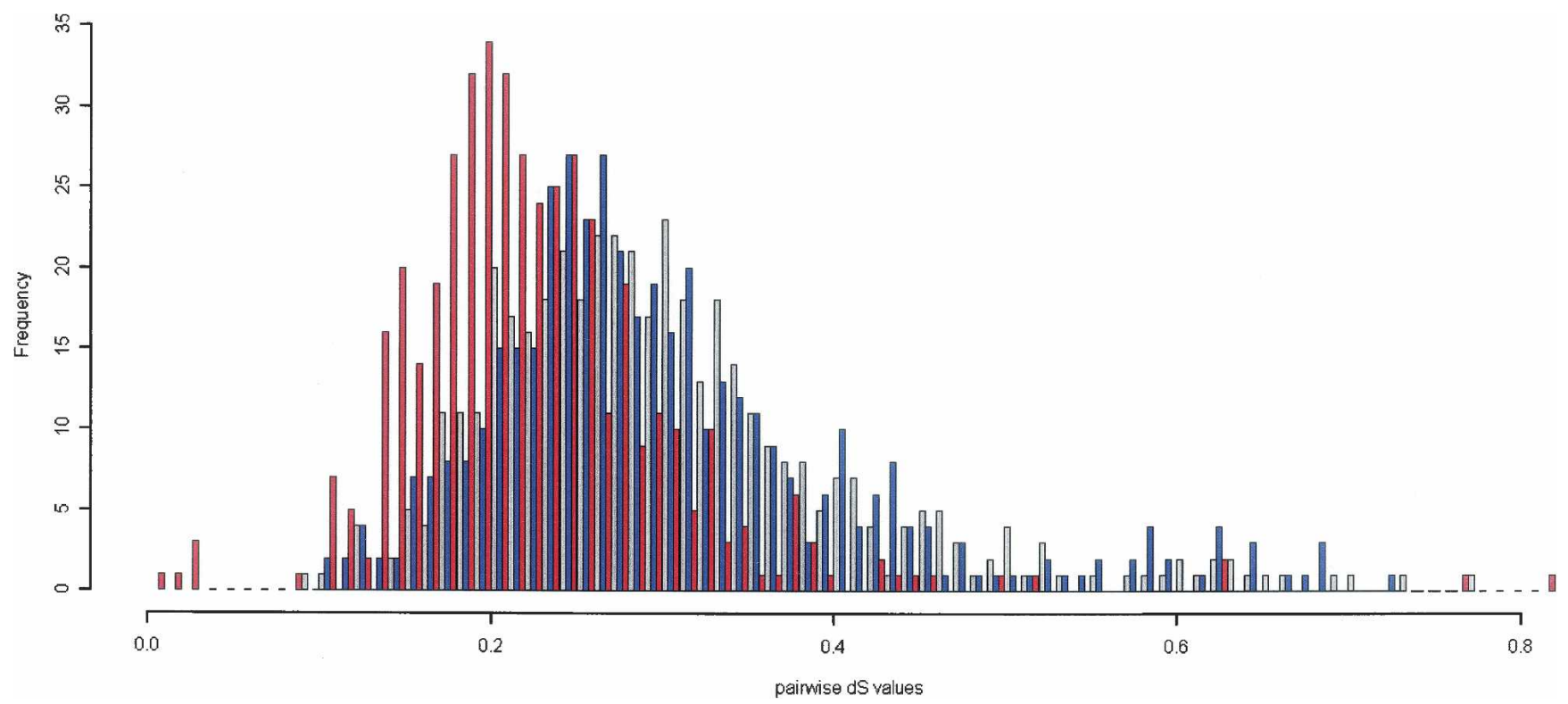

Figure 2. Distribution of $d_{\mathrm{S}}$ for pairwise comparisons between paralogs and orthologs. Distribution of $d_{\mathrm{S}}$ from pairwise comparison between $X$. laevis paralogs (red) and from pairwise comparisons between each $X$. laevis paralog from a trio with its $X$. tropicalis ortholog (blue and gray). The small number (eight in total) of $d_{\mathrm{s}}$ values that were $>1$ were eliminated to provide an appropriate scale. 
$\log$ (Table 2; Fig. 3; Supplemental Fig. S6), but we saw no significant enrichment of GO categories in the set of genes with retained paralogs. GO categories enriched in the single-copy genes, which have lost the tetraploidization-derived paralog, can be grouped into three main clusters related to respiration (cluster 1), nucleic acid processing (cluster 2), and nucleoside metabolism (cluster 3) (Table 2; Supplemental Fig. S5).

Next, we explored whether paralogous genes in $X$. laevis have begun to subfunctionalize at the level of gene expression. Subfunctionalized genes may retain similar or identical CDS while obtaining tissue-specific functions through mutations that alter their expression (Force et al. 1999). The UniGene project contains $\sim 27,000$ expressed sequence tags each from $X$. laevis and $X$. tropicalis that are derived exclusively from tissue-specific libraries (builds 63 and 24 respectively) (Pontius et al. 2003). Taking these tissue-specific ESTs, we matched each to its corresponding $X$. laevis and $X$. tropicalis gene where matches could be unambiguously assigned (BLASTN) (Altschul et al. 1997). For the 1039 X. laevis allotetraploidization-derived paralogs in our gene set (see Methods), 842 had EST matches in tissue-specific libraries for both clones. Of these, 118 (14.0\%) showed significant $(P<0.05$, corrected for multiple testing) differential tissue expression (Table 3; Supplemental Table S3) consistent with subfunctionalization. Next, we ranked $X$. laevis paralogs by the number of ESTs from any tissue in which each member of the pair is expressed. This approach gave a Spearman's rank correlation coefficient of 0.49 . A correlation of 1 would indicate that all paralogous gene pairs were similarly expressed, whereas a correlation near 0 would indicate that all gene pairs were expressed independently and randomly; the observed correlation of aggregate expression is intermediate to these extremes, which again is consistent with a degree of subfunctionalization in X. laevis. To assess how often paralogous genes have differential aggregate expression, we examined the highest and lowest deciles of aggregate expression. In the highest expression decile, $68 \%$ of genes have a paralog that is also in the highest expression decile, and in the lowest expression decile, $38 \%$ of genes have a paralog also in the lowest expression decile. This observation suggests that the function of both copies of highly expressed genes in $X$. laevis is more often conserved than is the function of sparsely expressed gene pairs.

\section{Discussion}

We report 10,967 full insert verified clones and sequences from $X$. laevis and $X$. tropicalis that have been generated by the XGC project. This clone set provides the community with a useful resource for functional genomic studies in these important model organisms. Furthermore, we have gained insight into the evolution of protein-coding sequences in a genome duplicated by species hybridization. It is well established that new genes typically arise through duplication of existing genes (Ohno 1970; Force et al. 1999). It is also known that whole genome duplications are common among the pipid frogs (Evans et al. 2004), and it is thought that these events are the major method of speciation within this genus (Kobel 1996; Evans et al. 2004). Because one member of a new duplicate gene pair is initially redundant, it is likely to quickly become a pseudogene after fixation of a null mutation within the population (Ohno 1970). The present study focuses on cDNA sequences; thus only actively transcribed genes (and potentially, rarely transcribed pseudogenes) contribute to our data set. For these expressed genes, the results of the present study indicate that many of the redundant copies have remained active in $X$. laevis over tens of millions of years. This result is consistent with previous observations in Xenopus, fish, and vari-

Table 2. GO Categories enriched in genes with no paralogs in $X$. laevis

\begin{tabular}{|c|c|c|c|c|c|}
\hline GO ID & Description & $\begin{array}{l}\text { Total genes } \\
\text { in category }\end{array}$ & $\begin{array}{l}\text { Genes in } \\
\text { category } \\
\text { with no } \\
\text { paralog }\end{array}$ & Enrichment & $\begin{array}{c}\text { False } \\
\text { discovery } \\
\text { rate }\end{array}$ \\
\hline GO:0006139 & $\begin{array}{l}\text { Nucleobase, nucleoside, nucleotide, and nucleic } \\
\text { acid metabolism }\end{array}$ & 446 & 125 & 1.450582 & 0 \\
\hline GO:0046112 & Nucleobase biosynthesis & 6 & 5 & 4.313063 & 0.0520 \\
\hline GO:0016070 & RNA metabolism & 108 & 34 & 1.629379 & 0.0533 \\
\hline GO:0006350 & Transcription & 85 & 28 & 1.704928 & 0.0571 \\
\hline GO:0006259 & DNA metabolism & 153 & 44 & 1.48843 & 0.0650 \\
\hline GO:0006082 & Organic acid metabolism & 116 & 35 & 1.561626 & 0.0600 \\
\hline GO:0019752 & Carboxylic acid metabolism & 116 & 35 & 1.561626 & 0.0600 \\
\hline GO:0009112 & Nucleobase metabolism & 7 & 5 & 3.696911 & 0.0717 \\
\hline GO:0006260 & DNA replication & 43 & 16 & 1.925833 & 0.0692 \\
\hline GO:0006099 & Tricarboxylic acid cycle & 10 & 6 & 3.105405 & 0.0600 \\
\hline GO:0009060 & Aerobic respiration & 10 & 6 & 3.105405 & 0.0600 \\
\hline GO:0045333 & Cellular respiration & 10 & 6 & 3.105405 & 0.0600 \\
\hline GO:0046356 & Acetyl-CoA catabolism & 10 & 6 & 3.105405 & 0.0600 \\
\hline GO:0051187 & Cofactor catabolism & 13 & 7 & 2.786902 & 0.0589 \\
\hline GO:0006206 & Pyrimidine base metabolism & 5 & 4 & 4.140541 & 0.0548 \\
\hline GO:0006207 & De novo pyrimidine base biosynthesis & 5 & 4 & 4.140541 & 0.0548 \\
\hline GO:0006544 & Glycine metabolism & 5 & 4 & 4.140541 & 0.0548 \\
\hline GO:0007223 & Frizzled-2 signaling pathway & 5 & 4 & 4.140541 & 0.0548 \\
\hline GO:0019856 & Pyrimidine base biosynthesis & 5 & 4 & 4.140541 & 0.0548 \\
\hline GO:0006396 & RNA processing & 68 & 22 & 1.674483 & 0.0579 \\
\hline GO:0006412 & Protein biosynthesis & 256 & 65 & 1.314136 & 0.0784 \\
\hline
\end{tabular}

All nonredundant Gene Ontology (Ashburner et al. 2000) terms enriched in the set of clones suspected to have no expressed paralogs in $X$. laevis. Categories with a false discovery rate (FDR) $\leq 0.10$ are shown. Several very large generic categories were removed for clarity. No statistically significant categories were found for genes for which a tetraploidization-derived paralog was found. 


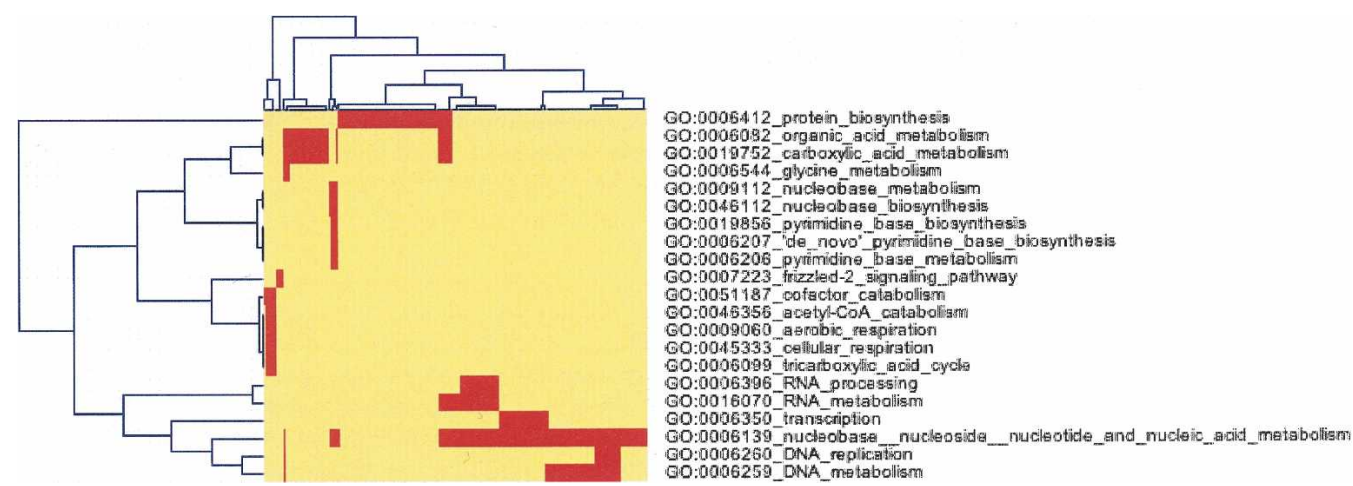

Figure 3. Clustered Image Map of genes with no paralog versus $\mathrm{GO}$ categories for categories with significant enrichment. Thumbnail clustered image map (CIM) of genes (top) versus categories (right) for categories with a false discovery rate (FDR) $\leq 0.10$. Very large generic categories have been removed to improve visualization. Clustering was performed with the Genesis Client (Sturn et al. 2002; http://genome.tugraz.at/Software/ GenesisCenter.html). Three major clusters can be seen. Processes involved in general metabolism (far left) include "cofactor catabolism," "acetyl-CoA catabolism," "aerobic respiration," "cellular respiration," and "tricarboxylic acid cycle." Processes involved in nucleic acid processing (bottom right) include "RNA metabolism," "transcription," "nucleobase metabolism," "DNA replication," and "DNA metabolism." The third cluster contains GO categories involved in nucleoside metabolism such as "nucleobase metabolism," "pyrimidine base biosynthesis," and "nucleobase biosynthesis." The full-size CIM in which all genes are displayed is available as Supplemental Figure S6.

ous plants (Hughes and Hughes 1993; Taylor et al. 2001; Adams and Wendel 2005).

Given that we observe 1039 paralog pairs after sampling only 8049 genes and using a conservative paralog assignment method, it is possible that retention of both copies within $X$. laevis has benefited the species. It has previously been estimated that $X$. laevis has retained about half of its duplicate genes (Bisbee et al. 1977). While there is no evidence for selective retention by functional category, we do provide evidence consistent with a model of selective loss of certain duplicate genes after genome duplication. Interestingly, the functional groups selectively lost in Xenopus, namely, respiration, nucleic acid processing, and nucleoside metabolism (Table 2; Supplemental Fig. S5), are included in those known to have been selectively lost after whole genome duplication in Arabidopsis thaliana (Maere et al. 2005). Genes in these categories, therefore, appear more likely than other genes to confer dosage sensitivity.

We searched for evidence of selection on the remaining tetraploidization-derived paralogs in $X$. laevis. Using the Yang and Nielsen maximum likelihood method (Yang 1997, 1998), we calculated pairwise $d_{\mathrm{N}} / d_{\mathrm{S}}$ ratios between tetraploidization-derived $X$. laevis paralogs and between each of these $X$. laevis paralogs and

Table 3. Differential tissue expression for $X$. laevis paralogs from EST information (top 20 genes)

\begin{tabular}{|c|c|c|c|c|c|c|c|}
\hline$P$-value & Gene 1 & Gene 2 & EST counts & Tissue & GO term & GO function & Best human BLAST hit \\
\hline $5.35 \mathrm{E}-138$ & BC072139 & BC088696 & 21 and 562 & Testes & GO:0006414 & Translational elongation & $\begin{array}{l}\text { Eukaryotic translation elongation } \\
\text { factor } 1 \delta\end{array}$ \\
\hline $1.06 \mathrm{E}-54$ & BC060381 & BC044961 & 263 and 21 & Testes & GO:0006826 & Iron ion transport & Ferritin, heavy polypeptide 1 \\
\hline $5.22 \mathrm{E}-23$ & BC054950 & BC056840 & 111 and 10 & Liver & GO:0006826 & Iron ion transport & Lactoferrin (copper form) \\
\hline $1.59 \mathrm{E}-15$ & BC054151 & BC041281 & 58 and 2 & Testes & GO:0003735 & Structural constituent of ribosome & Small ribosomal subunit \\
\hline $1.42 \mathrm{E}-10$ & BC046680 & BC057216 & 1 and 37 & Lung & GO:0006826 & Iron ion transport & Ferritin, heavy polypeptide 1 \\
\hline $9.31 \mathrm{E}-10$ & BC082829 & BC053760 & 30 and 0 & Heart & GO:0005509 & Calcium ion binding & Troponin C, slow \\
\hline $9.31 \mathrm{E}-10$ & BC077795 & BC072841 & 30 and 0 & Lung & GO:0005554 & Molecular function unknown & Hypothetical protein \\
\hline $1.42 \mathrm{E}-09$ & BC043895 & BC054956 & 2 and 37 & Testes & GO:0006412 & Protein biosynthesis & Ribosomal protein L4 \\
\hline 3.73E-09 & BC041282 & BC041307 & 28 and 0 & Testes & GO:0006412 & Protein biosynthesis & Ribosomal protein $\mathrm{S} 8$ \\
\hline 4.77E-07 & BC073285 & BC059975 & 21 and 0 & Kidney & GO:0005554 & Molecular function unknown & Latexin \\
\hline $1.37 \mathrm{E}-05$ & BC079989 & BC054174 & 3 and 25 & Brain & GO:0005554 & Molecular function unknown & No human hits \\
\hline $7.83 \mathrm{E}-05$ & BC043906 & ВС077529 & 22 and 3 & Ovary & GO:0006810 & Transport & Migration-inducing protein MIG8 \\
\hline 0.000122 & BC070531 & BC044682 & 13 and 0 & Kidney & GO:0006839 & Mitochondrial transport & Uncoupling protein homolog \\
\hline 0.000259 & BC092102 & BC072833 & 1 and 15 & Testes & GO:0005554 & Molecular function unknown & $\begin{array}{l}\text { Thioredoxin-dependent peroxide } \\
\text { reductase } 2\end{array}$ \\
\hline 0.000428 & BC072304 & BC056053 & 3 and 19 & Heart & GO:0018149 & Peptide cross-linking & Uncoupling protein-2 \\
\hline 0.000462 & BC041242 & BC073375 & 31 and 64 & Testes & GO:0006412 & Protein biosynthesis & Ribosomal protein $\$ 9$ \\
\hline 0.000488 & BC081147 & BC072297 & 11 and 0 & Testes & GO:0005554 & Molecular function unknown & $\begin{array}{l}\text { ADP-ribosylhydrolase like } 1 \text { isoform } \\
1\end{array}$ \\
\hline 0.000488 & BC045043 & ВС073411 & 11 and 0 & Testes & GO:0005554 & Molecular function unknown & WD40 protein Ciao1 variant \\
\hline 0.000488 & BC078599 & BC074203 & 11 and 0 & Testes & GO:0006334 & Nucleosome assembly & H2A histone family, member Q \\
\hline 0.000488 & BC059972 & BC044112 & 11 and 0 & Heart & GO:0006468 & Protein amino acid phosphorylation & $\begin{array}{l}\text { Pyruvate dehydrogenase kinase, } \\
\text { isoenzyme } 4\end{array}$ \\
\hline
\end{tabular}

A summary of the top 20 paralog pairs with evidence for subfunctionalization (sorted with most significant $P$-value at the top). The EST count for gene 1 and gene 2 of each paralog pair in the tissue showing differential expression is shown. The Gene Ontology (GO) category (Ashburner et al. 2000) that best describes the putative function of each gene pair is included in addition to the name of the best Homo sapiens BLASTP hit. All 118 cases of potential subfunctionalization are supplied in Supplemental Table S3.

\section{Genome Research}

www.genome.org 
its $X$. tropicalis ortholog. While none of the paralog pairs observed in this study provides direct evidence of positive Darwinian selection, it must be noted that the evolutionary distance between $X$. laevis and $X$. tropicalis is approaching the practical limit of detecting positive selection by the $d_{\mathrm{N}} / d_{\mathrm{S}}$ test (Hughes et al. 2000). Also, all $d_{\mathrm{N}} / d_{\mathrm{S}}$ ratios are averages across the length of each gene, and may mask situations in which positive selection has only occurred in a site-specific manner. Interestingly, overall, $d_{\mathrm{N}} / d_{\mathrm{S}}$ ratios between tetraploidization-derived $X$. laevis paralogs were significantly elevated relative to $d_{\mathrm{N}} / d_{\mathrm{S}}$ ratios between $X$. laevis/X. tropicalis orthologs (Fig. 1). This result is consistent with the notion that gene duplication has, to a small but measurable degree, freed expressed $X$. laevis sequence from functional constraint.

A subset of $X$. laevis paralogs also shows strong evidence for subfunctionalization, as indicated by the differential expression observed between $\sim 14 \%$ of the paralog pairs analyzed. The paralogs that show differential expression at the highest significance levels are overrepresented in a few molecular functions (Table 3). In the top 20 subfunctionalized pairs, five are involved in protein translation, and five are potentially involved in either iron or calcium transport. With the application of Spearman's rank to paralog pairs, we have detected a global trend of many of the paralog pairs toward subfunctionalization. The observation of numerous examples in which two allotetraploidization-derived gene copies show differential expression lends support to the theory of regulatory evolution (King and Wilson 1975), which holds that evolution is mediated by modification of gene expression patterns as well as by coding sequence changes.

\section{Methods}

The analyses described here were performed on the 10,967 Xenopus sequences available from the XGC Web site as of September 9, 2005 (XGC homepage, http://xgc.nci.nih.gov/).

\section{Selection of clones for sequencing}

Candidate clones for full insert sequencing were selected as previously described (Gerhard et al. 2004). Identification of additional putatively novel Xenopus clones was as follows. Pairs of orthologous (in the reciprocal best-match sense) $X$. tropicalis and $X$. laevis sequences were aligned, and the $5^{\prime}$-most ATG in the sequence was used to divide the alignment. Then 674 pairs of characterized genes were examined to determine mean conservation in CDS and 5'-UTR (92.9\% and $89.7 \%$, respectively), as well as to compute the variance of the conservation difference between CDS and UTRs. Sequences showing any increase in conservation (corresponding to a sequence conservation of 1.6 standard deviations below the mean) and with a 5 '-UTR of at least 25 nt were selected for sequencing.

\section{Paralog and ortholog assignment}

For each species, an all-by-all BLASTN search was executed, and any sequences with $>98 \%$ nucleic acid identity over $90 \%$ of their length were removed as redundant copies of the same clone, allelic copies, or recent gene duplicates. Next, unmatched sequences from the $X$. laevis reciprocal BLASTN and those with more than one significant $\left(e<10^{-20}\right)$ match were excluded, leaving 1354 putative allotetraploidization-derived $X$. laevis paralogs with $93.1 \% \pm 2.72 \%$ nucleic acid identity (mean \pm SD). Finally, a reciprocal BLASTP search of each $X$. laevis paralogous against the set of $X$. tropicalis predicted nonredundant proteins (EnsEMBL homepage, http://www.ensembl.org/Xenopus tropicalis) identified 1039 paralog pairs for which both clones had a common best $X$. tropicalis hit. Note that the above approach automatically prevents us from considering gene families with more than 1:1 paralog mapping and prevents assignment of tetraploidization-derived paralogs to those that formed prior to the speciation between $X$. laevis and $X$. tropicalis. Also, by considering the best nonredundant match of each $X$. laevis clone sequence, we assigned the most recent paralog pairs, assuming that few paralog pairs have formed since the tetraploidization event. Of the 1039 gene trios, 445 have an ortholog that is represented in the set of $2918 X$. tropicalis full ORF cDNAs. Our $d_{\mathrm{N}} / d_{\mathrm{S}}$ analysis is based on these 445 trios, and we included the additional paralog pairs with no $X$. tropicalis XGC ortholog in the retained paralog and subfunctionalization analyses.

\section{Multiple sequence alignments}

We performed all protein alignments using CLUSTALW with default parameters (Thompson et al. 1994). We used the RevTrans program (Wernersson and Pedersen 2003) to produce codonaware alignments for more accurate predictions of substitution rates. Files were formatted from RevTrans output (FASTA) to codeml input format with a Perl script.

\section{$d_{\mathrm{N}} / d_{\mathrm{S}}$ estimation}

We performed pairwise $d_{\mathrm{N}}, d_{\mathrm{S}}$ and $\omega$ calculations using the yn00 component of the PAML package. We also calculated $d_{\mathrm{N}} / d_{\mathrm{S}}$ with the maximum likelihood method of the codeml program (Yang et al. 1997) in order to perform likelihood ratio tests. Each $d_{N} / d_{\mathrm{S}}$ calculation was performed twice. In one run, the $d_{\mathrm{N}} / d_{\mathrm{S}}$ was fixed at 1 , and in the other run it was free to vary. Twice the difference between the two log likelihood values was compared to the $\chi^{2}$ distribution with 1 degree of freedom for rejection of the hypothesis that $d_{\mathrm{N}} / d_{\mathrm{S}}=1(P<0.05)$.

\section{Gene Ontology analysis of retained paralogs}

The aim of this analysis was to determine whether genes of specific functions have been selectively retained in multiple copies within the $X$. laevis genome or whether redundant copies have been selectively lost after duplication. Clones with no expressed paralog were defined by first assigning a UniGene cluster to each clone as described above. We limited our analysis to clones for which this corresponding UniGene cluster contained at least 20 ESTs to minimize the potential of assigning genes with low expression as single-copy genes. Single-copy genes were then assigned by the absence of a second significant $\left(e<10^{-30}\right)$ BLASTN hit in UniGene. We searched for GO categories enriched in either the 1527 single-copy genes or the 1039 dual-copy genes using High-Throughput GoMiner (http://discover.nci.nih.gov/ gominer/htgm.jsp) (Zeeberg et al. 2005) and an in-house customized version of the GO Consortium (Ashburner et al. 2000) database. This database was populated with all GO annotations assigned by Interproscan (Apweller et al. 2001) to the X. laevis genes. The statistical significance of enrichment was based on a false discover rate (FDR) threshold value of 0.10, using HighThroughput GoMiner's correction for multiple testing.

\section{Tissue expression comparisons}

We matched every clone to its best UniGene cluster by a BLASTN of each clone against a representative EST from each UniGene cluster in the files Xl.seq.uniq and Str.seq.uniq downloaded from NCBI (UniGene Download page, ftp://ftp.ncbi.nih.gov/ 
repository/UniGene). We then performed a second BLASTN search of each clone against all ESTs in its representative cluster and only considered matches with alignment lengths $>200 \mathrm{bp}$ and percent identities $>90 \%$. As some $(20)$ of the genes shared a common UniGene cluster, we only used ESTs from those clusters if they could be unambiguously assigned to one copy by differential percent identity. In all other cases, all reads from the best UniGene cluster for each clone were used. Using the library name for each EST, we determined the source tissue of the EST as defined in the files Xl.lib.info and Str.lib.info. A Perl script counted the total number of ESTs of each gene found in each tissue. Using a previously described Bayesian method (Audic and Claverie 1997), we assigned $P$-values to each gene pair showing a significant difference in expression $(P<0.05)$. As the variability in expression patterns was strongest in tissues where the genes are highly expressed, we chose to only compare the expression of the two paralogs in the tissue in which they showed to be most highly expressed (highest EST count). A Bonferroni correction was used to compensate for multiple hypothesis testing where applicable, although a maximum of two tests were done for each paralog pair.

\section{Acknowledgments}

This project has been funded in part with Federal funds from the National Human Genome Research Institute, National Institutes of Health, under contract No. U01 HG002155-06S1. This research was supported (in part) by the Intramural Research Program of the National Cancer Institute (NCI) of the National Institutes of Health (NIH). This work would also not have been possible without the cDNA libraries and EST sequences provided by various groups and we specifically thank Igor Dawid and Thomas Sargent (NICHD), Donald Brown (Carnegie Institute), Bruce Blumberg (UC at Irvine), and Robert Grainger (UVA). Work by C.P.P. was performed under the auspices of the U.S. Department of Energy by the University of California, Lawrence Livermore National Laboratory under contract No. W-7405-Eng-48. We thank Diana Palmquist and Elizabeth Chun, who assisted with finishing some of the clones. R.A.H., S.J.M.J., and M.A.M. are Michael Smith Foundation for Health Research Scholars.

\section{References}

Adams, K.L. and Wendel, J.F. 2005. Polyploidy and genome evolution in plants. Curr. Opin. Plant Biol. 8: 135-141.

Altschul, S.F., Madden, T.L., Schaffer, A.A., Zhang, J., Zhang, Z., Miller, W., and Lipman, D. 1997. Gapped BLAST and PSI-BLAST: A new generation of protein database search programs. Nucleic Acids Res. 25: 3389-3402.

Apweller, R., Attwood, T.K., Bairoch, A., Bateman, A., Birney, E., Biswas, M., Bucher, P., Cerutti, L., Corpet, F., Croning, M.D.R., et al. 2001. The InterPro database, an integrated documentation resource for protein families, domains and functional sites. Nucleic Acids Res. 29: 37-40.

Ashburner, M., Ball, C.A., Blake, J.A., Botstein, D., Butler, H., Cherry, J.M., Davis, A.P., Dolinski, K., Dwight, S.S., Eppig, J.T., et al. The Gene Ontology Consortium. 2000. Gene Ontology: Tool for the unification of biology. Nat. Genet. 25: 25-29.

Audic, S. and Claverie, J.M. 1997. The significance of digital gene expression profiles. Genome Res. 7: 986-995.

Bisbee, C.A., Baker, M.A., Wilson, A.C., Irandokht, H.A., and Fischberg, M. 1977. Albumin phylogeny for clawed frogs. Science 195: 785787.

Blackshear, P.J., Lai, W.S., Thorn, J.M., Kennington, E.A., Staffa, N.G., Moore, D.T., Bouffard, G.G., Beckstrom-Sterberg, S.M., Touchman, J.W., de Fatima Bonaldo, M., et al. 2001. The NIEHS Xenopus maternal EST project: Interim analysis of the first 13,879 ESTs from unfertilized eggs. Gene 267: 71-87.
Butterfield, Y.S., Marra, M.A., Asano, J.K., Chan, S.Y., Guin, R., Krzywinski, M.I., Lee, S.S., MacDonald, K.W., Mathewson, C.A., Olson, T.E., et al. 2002. An efficient strategy for large-scale high-throughput transposon-mediated sequencing of cDNA clones. Nucleic Acids Res. 30: 2460-2468.

De Sa, R.O. and Hillis, D.M. 1990. Phylogenetic relationships of the pipid frogs Xenopus and Silurana: An integration of ribosomal DNA morphology. Mol. Biol. Evol. 7: 365-376.

Evans, B.J., Kelley, D.B., Tinsley, R.C., Melnick, D.J., and Cannatella, D.C. 2004. A mitochondrial DNA phylogeny of African clawed frogs: Phylogeography and implications for polyploid evolution. Mol. Phylogenet. Evol. 33: 197-213.

Ewing, B. and Green, P. 1998. Base-calling of automated sequencer traces using Phred. II. Error probabilities. Genome Res. 8: 186194.

Force, A., Lynch, M., Pickett, F.B., Amores, A., Yan, Y., and Postlethwait, J. 1999. Preservation of duplicate genes by complementary, degenerative mutations. Genetics 151: 1531-1545.

Gerhard, D.S., Wagner, L., Feingold, E.A., Shenmen, C.M., Grouse, L.H., Schuler, G., Klein, S.L., Old, S., Rasooly, R., Good, P., et al. 2004. The status, quality, and expansion of the NIH Full-Length cDNA Project: The Mammalian Gene Collection (MGC). Genome Res. 14: $2121-2127$.

Gilchrist, M.J., Zorn, A.M., Voigt, J., Smith, J.C., Papalopulu, N., and Amaya, E. 2004. Defining a large set of full-length clones from a Xenopus tropicalis EST project. Dev. Biol. 271: 498-516.

Gordon, D., Abajian, C., and Green, P. 1998. Consed: A graphical tool for sequence finishing. Genome Res. 8: 195-202.

Graf, J. and Kobel, H. 1991. Genetics of Xenopus laevis. Methods Cell Biol. 36: 663-669.

Hirsch, N., Zimmerman, L., and Grainger, R. 2002. Xenopus, the next generation: X. tropicalis genetics and genomics. Dev. Dyn. 225: 422-433.

Hughes, M.K. and Hughes, A.L. 1993. Evolution of duplicate genes in a tetraploid animal, Xenopus laevis. Mol. Biol. Evol. 10: 1360-1369.

Hughes, M.K., Green, J.A., Garbayo, J.M., and Roberts, R.M. 2000. Adaptive diversification within a large family of recently duplicated, placentally expressed genes. Proc. Natl. Acad. Sci. 97: 3319-3323.

King, M.C. and Wilson, A.C. 1975. Evolution at two levels in humans and chimpanzees. Science 188: 107-116.

Klein, S.L., Strausberg, R.L., Wagner, L., Pontius, J., Clifton, S.W., and Richardson, P. 2002. Genetic and genomic tools for Xenopus research: The NIH Xenopus Initiative. Dev. Dyn. 225: 384-391.

Kobel, H.R. 1996. Allopolyploid speciation. In The biology of Xenopus (eds. R.C. Tinsley and H.R. Kobel), pp. 391-401. Clarendon Press, Oxford.

Maere, S., De Bodt, S., Raes, J., Casneuf, T., Van Montagu, M., Kuiper, M., and Van de Peer, Y. 2005. Modeling gene and genome duplication in eukaryotes. Proc. Natl. Acad. Sci. 102: 5454-5459.

Ohno, S. 1970. Evolution by gene duplication. Springer-Verlag, New York.

Pontius, J.U., Wagner, L., and Schuler, G.D. 2003. UniGene: A unified view of the transcriptome. In The NCBI Handbook, pp. 857-868. National Center for Biotechnology Information, Bethesda, MD.

Strausberg, R.L., Feingold, E.A., Grouse, L.H., Derge, J.G., Klausner, R.D. Collins, F.S., Wagner, L., Shenmen, C.M., Schuler, G.D., and Altschul, S.F. 2002. Generation and initial analysis of more than 15,000 full-length human and mouse cDNA sequences. Proc. Natl. Acad. Sci. 99: 16899-16903.

Sturn, A., Quackenbush, J., and Trajanoski, Z. 2002. Genesis: Cluster analysis of microarray data. Bioinformatics 18: 207-208.

Taylor, J.S., Van de Peer, Y., Braasch, I., and Myer, A. 2001. Comparative genomics provides evidence for an ancient genome duplication event in fish. Philos. Trans. R. Soc. Lond. B Biol. Sci. 356: 1661-1679.

Thompson, J.D., Higgins, D.G., and Gibson, T.J. 1994. CLUSTAL W: Improving the sensitivity of progressive multiple sequence alignment through sequence weighting, position specific gap penalties and weight matrix choice. Nucleic Acids Res. 22: 4673-4680.

Thornton, K. and Long, M. 2002. Rapid divergence of gene duplicates on the Drosophila melanogaster X chromosome. Mol. Biol. Evol. 19: 918-925.

Wernersson, R. and Pedersen, A.G. 2003. RevTrans: Multiple alignment of coding DNA from aligned amino acid sequences. Nucleic Acids Res. 31: $3537-3539$.

Wilson, R.K. and Mardis, E.R. 1997. Fluorescence-based DNA sequencing. In Genome analysis: A laboratory manual: Analyzing DNA (eds. B. Birren et al.), Vol. 1, pp. 301-395. Cold Spring Harbor Laboratory Press, Cold Spring Harbor, NY.

Yang, Z. 1997. PAML, a program package for phylogenetic analysis by maximum likelihood. Comput. Appl. Biosci. 13: 555-556.

. 1998. Likelihood ratio tests for detecting positive selection and

\section{Genome Research}

www.genome.org 


\section{0,967 full-length cDNA clones from Xenopus}

application to primate lysozyme evolution. Mol. Biol. Evol. 15: $568-573$.

Yang, G., Stott, J.M., Smailus, D.M., Barber, S.A., Balasundaram, M., Marra, M.A., and Holt, R.A. 2005. High-throughput sequencing: A failure mode analysis. BMC Genomics 6: 2 .

Zeeberg, B.R., Qin, H., Narasimhan, S., Sunshine, M., Cao, H., Kane,

D.W., Reimers, M., Stephens, R.M., Bryant, D., Burt, S.K., et al. 2005.

High-Throughput GoMiner, an 'industrial-strength' integrative gene ontology tool for interpretation of multiple-microarray experiments, with application to studies of Common Variable Immune Deficiency (CVID). BMC Bioinformatics 6: 168.

Zhang, L., Vision, T., and Gaut, B. 2002. Patterns of nucleotide substitution among simultaneously duplicated gene pairs in Arabidopsis thaliana. Mol. Biol. Evol. 19: 1464-1473.

Received October 28, 2005; accepted in revised form March 16, 2006. 
Genome Research 16: 796-803 (2006)

\section{Sequencing and analysis of 10,967 full-length cDNA clones from Xenopus laevis and Xenopus tropicalis reveals post-tetraploidization transcriptome remodeling}

Ryan D. Morin, Elbert Chang, Anca Petrescu, Nancy Liao, Malachi Griffith, Robert Kirkpatrick, Yaron S. Butterfield, Alice C. Young, Jeffrey Stott, Sarah Barber, Ryan Babakaiff, Mark C. Dickson, Corey Matsuo, David Wong, George S. Yang, Duane E. Smailus, Keith D. Wetherby, Peggy N. Kwong, Jane Grimwood, Charles P. Brinkley III, Mabel Brown-John, Natalie D. Reddix-Dugue, Michael Mayo, Jeremy Schmutz, Jaclyn Beland, Morgan Park, Susan Gibson, Teika Olson, Gerard G. Bouffard, Miranda Tsai, Ruth Featherstone, Steve Chand, Asim S. Siddiqui, Wonhee Jang, Ed Lee, Steven L. Klein, Robert W. Blakesley, Barry R. Zeeberg, Sudarshan Narasimhan, John N. Weinstein, Christa Prange Pennacchio, Richard M. Myers, Eric D. Green, Lukas Wagner, Daniela S. Gerhard, Marco A. Marra, Steven J.M. Jones, and Robert A. Holt

One of the authors was inadvertently left off of this list. William Chow should be added after Malachi Griffith. His affiliation is British Columbia Genome Sciences Centre, BCCA, Vancouver, BC V5Z 1L3 Canada. The authors apologize for this omission.

Genome Research 16: 713-722 (2006)

\section{Evolutionary turnover of mammalian transcription start sites}

Martin C. Frith, Jasmina Ponjavic, David Fredman, Chikatoshi Kai, Jun Kawai, Piero Carninci, Yoshihide Hayshizaki, and Albin Sandelin

One of the authors' names was inadvertently misspelled. Yoshihide Hayshizaki should be Yoshihide Hayashizaki. 


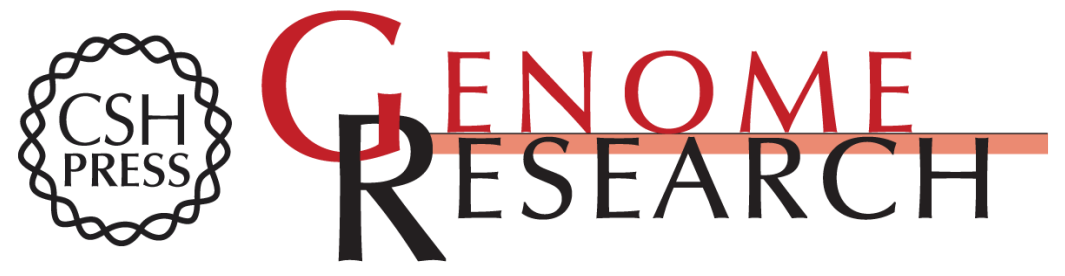

\section{Sequencing and analysis of 10,967 full-length cDNA clones from Xenopus laevis and Xenopus tropicalis reveals post-tetraploidization transcriptome remodeling}

Ryan D. Morin, Elbert Chang, Anca Petrescu, et al.

Genome Res. 2006 16: 796-803

Access the most recent version at doi:10.1101/gr.4871006

\footnotetext{
Supplemental http://genome.cshlp.org/content/suppl/2006/05/03/gr.4871006.DC1 Material

Related Content Erratum

Genome Res. July , 2006 16: 947

References This article cites 33 articles, 9 of which can be accessed free at: http://genome.cshlp.org/content/16/6/796.full.html\#ref-list-1

Articles cited in:

http://genome.cshlp.org/content/16/6/796.full.html\#related-urls

License
}

Email Alerting Receive free email alerts when new articles cite this article - sign up in the box at the Service top right corner of the article or click here.

\section{Affordable, Accurate Sequencing.}

